\title{
Towards an Ab-initio Characterization of Novel Intermediate Band Photovoltaic Materials
}

\author{
P. Wahnón, P. Palacios, I. Aguilera and K. Sánchez
}

\begin{abstract}
An ab-initio study of novel photovoltaic materials with enhanced optoelectronic properties is presented in this contribution. Predictions of absorption coefficients agree completely with the characterization of the first experimental samples grown in the laboratory. Compounds selected for the study are derived from chalcogenide semiconductors in which several atoms are substituted by transition elements. These atoms modify the electronic band structure in such a way that a new narrow band appears inside the parent semiconductor band-gap. Partial occupation of this band enables that additional carriers could be obtained from absorption of photons with energy lower than that of the band-gap, thus enhancing the photovoltaic conversion properties of the material. It was estimated than a photovoltaic solar cell designed from this novel concept could reach a thermodynamic efficiency of $63.2 \%$ compared to $43.1 \%$ corresponding to the thermodynamic efficiency limit of conventional semiconductor based solar cells. Results show a significant enhancement of the absorption coefficient respecting to the corresponding parent semiconductor in the main emission region of the solar spectrum. For some of the theoretically proposed compounds, optoelectronic properties of recently synthesized samples have been obtained experimentally. Comparison of experimental absorption measurements with results of the calculations presented here shows a very good agreement.
\end{abstract}

Chalcopyrites, spinels, Solar cells, ab-initio

\section{INTRODUCTION}

The Intermediate Band (IB) photovoltaic material concept was postulated to be able to enhance the efficiency of solar cells [1]. In the last years our group has presented several materials $[2-5]$ as candidate systems whose properties fulfill the IB material concept. Our proposal is based on the substitution of some atoms in selected semiconductors by several transition metals which introduce a new band inside the host material band-gap. The first proposed candidates to obtain this intermediate band were transition metal-doped III-V materials based on GaAs or GaP [1]. In these compounds the substituted atom was the Gallium.

The IB band must be partially filled and energetically isolated from the semiconductor gap between the valence (VB) and conduction (CB) band. This proposal will take advantage of a larger fraction of the solar spectrum than common one gap cells and will allow increasing the photocurrent without decreasing the photovoltage. As usual in semiconductors an electron can be excited from the VB to the CB but, now in these alloys we will have also two more electron transitions: first there is an excitation from the VB to the IB, and second, from the IB to the CB. These lower energy transitions will be the responsible of the light absorption enhancement. The IB concept has an ideal solar energy conversion limit of up to $63.2 \%$. Next proposed compound family to have an intermediate band was chalcopyrite $\left(\mathrm{CuGaS}_{2}\right)$. Both families (III-V and chalcopyrites) were computed at different dilution levels and with metallic substitutions at several sites. The $3 \mathrm{~d}$ metal substitutes were Titanium, Vanadium, Chromium and Manganese. The metals are in a tetrahedral environment around them and they are in $\mathrm{a}^{3+}$ oxidation state. Their $3 \mathrm{~d}$ states are partially filled and splitted in two manifolds according to the aforementioned coordination.

All these studies have been made theoretically using the Density Functional Theory (DFT) including spin polarization and using the local density (LDA) and the generalized gradient (GGA) approximation. In this work we have calculated band diagrams and electronic densities of states projecting selected atoms. Dielectric function was also calculated from converged wave functions. This allows us to obtain some optical properties. We will also identify which transitions corresponds to each peak in the absorption coefficient using a projection of the imaginary part of the dielectric function. Previously we have to include in our calculation the relaxation of forces and stresses to predict the structure of the novel material.

The aim of this work is to continue with the study of chalcopyrites and to introduce a new family of systems which are indium-based thio-spinels which will be again substituted by transition metal atoms [6]. Both are chalcogenide semiconductors which are well known and spreadly used in the photovoltaic field, often as thin films solar cell materials or window layers. The main difference in thio-spinel substituted systems compared with the chalcopyrite ones is that the environment around the metal atom is not tetrahedral but octahedral coordinated, so we will have six atoms around the metal. This new coordination changes the splitting of metal $3 \mathrm{~d}$ orbitals. In the case of chalcopyrite based structure substituted by $\mathrm{Ti}$ the intermediate band correspond to the low-energy $\mathrm{e}_{\mathrm{g}}$ doublet split from the $3 \mathrm{~d}$ shell in a tetrahedral environment, while for the spinel based compounds taking $\mathrm{Ti}$ or $\mathrm{V}$ as substitute the intermediate band comes from the low-energy $t_{2 g}$ triplet originated from the splitting of the $3 d$ shell in an octahedral crystalline field. This new orientation must be thermodynamically more favorable than the tetrahedral coordination approach because these metal atoms in a $\mathrm{M}^{3+}$ oxidation state prefer to be coordinated in that way. 


\section{MODELS}

I-III-VI $\mathrm{VI}_{2}$ type chalcopyrite semiconductors crystallize in the I-42d space group, number 122, with four formula units in each tetragonal centered unit cell. It is a superstructure of zincblende, distorted in the $\mathrm{z}$ direction, where each atom is tetrahedral coordinated to four neighboring atoms. The resulting structure after the Gallium substitution by a transition metal has 16 atoms in its unit cell. The final chemical formula is $\mathrm{Cu}_{4} \mathrm{Ga}_{3} \mathrm{~S}_{8} \mathrm{M}$.

Spinels are a family of compounds whose chemical formula is $\mathrm{II}-\mathrm{III}_{2}-\mathrm{VI}_{4}$ which crystallizes in $\mathrm{Fd} 3 \mathrm{~m}$ space group. In their structures, anions form a face centered cubic lattice, surrounded by tetrahedral and octahedral sites which are occupied by the cations. Spinels can be classified as direct or inverse. In direct spinels, II type atoms are on tetrahedral sites and the Ill-type atoms are on octahedral sites. For the inverse compounds the II-type atoms change their positions and become to be in octahedral sites. Experimentally the $\operatorname{MgIn}_{2} \mathrm{~S}_{4}$ spinel has some degree of inversion but we have studied its direct structure for simplicity. The studied spinel derivative was obtained by substitution of one Indium by a transition metal (Titanium or Vanadium) resulting a $\mathrm{Mg}_{2} \mathrm{In}_{3} \mathrm{~S}_{8} \mathrm{M}$ final formula.

The $\mathrm{In}_{2} \mathrm{~S}_{3}\left(\mathrm{In}_{16} \mathrm{~S}_{24}\right.$ unit cell) system can be considered as a defective structure of the above $\mathrm{Mg}_{2} \mathrm{In}_{4} \mathrm{~S}_{8}$ spinel with $\mathrm{Mg}$ vacancies. The intermediate band system has been made by substitution of Indiums at octahedral sites by a transition metal and has a $\operatorname{In}_{14} \mathrm{~S}_{24} \mathrm{M}_{2}$ formula. The crystal structures of the aforementioned systems are shown in Fig 1.
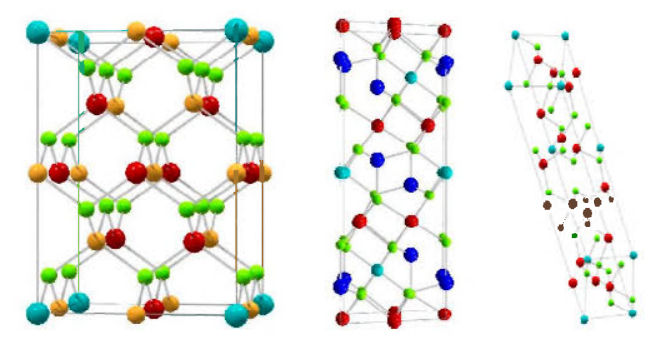

Fig1. Cu4Ga3S8M (left), Mg2In3S8M (center) and In14S24M2 (right) crystal structure, being $\mathrm{M}$ a metal $=\mathrm{Ti}$ or $\mathrm{V}$.

\section{METHODS}

We have made spin-polarized DFT calculations mainly at the Perdew and Wang functional (PW91) level using the planewave VASP program with PAW potentials [7]. Convergence tests of kinetic cutoff and k-points sampling were done independently for all the systems until they were satisfactorily converged. Unconstrained atomic relaxations were made with convergence tolerance of $0.01 \mathrm{eV} / \mathrm{Angs}$ for atomic forces for all the systems.

Concerning the calculation of the optical properties we have used the so-called Random Phase Approximation (RPA) consisting in obtaining the imaginary part of the dielectric function (and so other optical properties) from the wavefunctions, eigenvalues, and occupations of the electronic states that were previously calculated. The general method to obtain these properties is described below. We can write the elements of the imaginary part of the dielectric tensors as a sum over occupied and unoccupied states as in (1).

$$
\begin{array}{r}
d_{n \beta \beta}^{(2)}(\omega)=\frac{4 \pi^{2} e^{2}}{\Omega m^{2} \omega^{2}} \sum_{k n n^{\prime} \sigma} f_{n \sigma}^{k}\left(1-f_{n^{\prime} \sigma}^{k}\right) \delta\left(\xi_{k n^{\prime}}-\xi_{k n}-\hbar \omega\right) \\
x\left\langle\psi_{n k \sigma}\left|p_{\alpha}\right| \psi_{n^{\prime} k \sigma}\right\rangle\left\langle\psi_{n^{\prime} k \sigma}\left|p_{\beta}\right| \psi_{n k \sigma}\right\rangle
\end{array}
$$

where $\psi_{n k \sigma}$ and $\psi_{n^{\prime} k \sigma}$ are the crystal wavefunctions corresponding to the initial and final states at the k-point $\mathrm{k}$. Respectively, $e, m$ and $\Omega$ are the electron charge, its mass and the volume of the unit cell, and $f_{i t w}^{\bar{k}}$ is the occupation of the level, according to the Fermi distribution.

With this, we can compute the real part of the dielectric function by the Kramers-Kronig equations (2), and with both of them we will obtain the fundamental optical properties; such as absorption, reflectance, refraction index, etc.; by simple relations. More details of this method can be found in reference [8].

$$
\varepsilon_{\alpha \beta}^{(1)}(\omega)=1+\frac{2}{\pi} \wp \int \frac{\omega^{\prime}}{\omega^{\prime 2}-\omega^{2}+\eta \eta} \varepsilon_{\alpha \beta}^{(2)}\left(\omega^{\prime}\right) d \omega^{\prime}
$$

We use (1) and (2) as implemented in the post-processing tool of VASP called OPTIC [9]. For these calculations we again tested the k-point sampling until convergence was found.

\section{RESULTS}

\section{A .Band Structure Calculations}

Previously to obtain optoelectronic properties is necessary to calculate the ground-state properties for all the systems. First we have compared the results for the semiconductors with available experimental and theoretical data $[10,11]$. The geometrical results compared very well with them. The main difference appears in the band-gap energy which is underestimated by our results compared to experiment but agrees with other theoretical calculations. This is a general problem of the DFT method. After that we have made calculations in the substituted systems. When a Gallium atom in the chalcopyrite or an Indium atom in the spinel is substituted by some transition atoms, an intermediate band appears at the spin-up channel. In Fig 2 we show the spin-up total and metal-projected density of states diagram for three proposed intermediate band systems and the metal projection. In the projections, we can clearly see that the intermediate band is mainly due to the transition metal introduced in the semiconductor system.

\section{B. Optical properties calculation.}

The optical absorption coefficient has been calculated for the semiconductor as well as for the substituted alloys. In order to understand the origin of absorption peaks in the spectrum and to interpret which transitions contribute to each one, we calculated the frequency-weighted imaginary part of the total dielectric function and its partial contribution to the different transitions. The peaks for this magnitude appear at the same energies than absorption peaks but they present different 
amplitudes. We consider this is a good way to understand the intermediate band contribution to the global absorption.
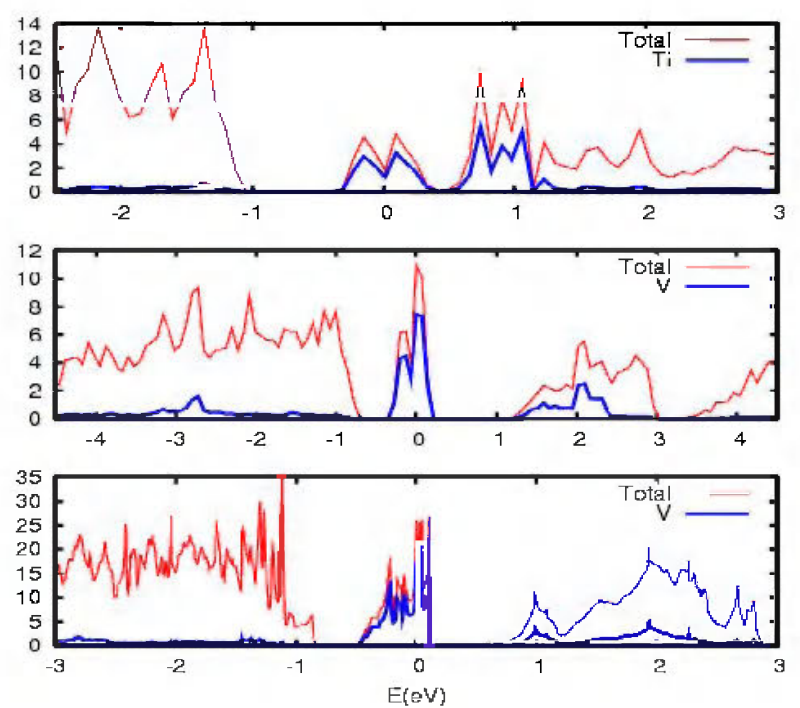

Fig 2 GGA spin-up total DOS and metal-projected DOS for the $\mathrm{Cu}_{4} \mathrm{Ga}_{3} \mathrm{~S}_{8} \mathrm{Ti}$ (top), $\mathrm{Mg}_{2} \mathrm{In}_{3} \mathrm{~S}_{8} \mathrm{~V}$ (center) and $\operatorname{In}_{14} \mathrm{~S}_{24} \mathrm{~V}_{2}$ (bottom) substituted systems.

l) CuGaS2 and derivatives: Fig 3 show the effect of the $\mathrm{M}=\mathrm{Ti}$. $\mathrm{Cr}$ substitution in $\mathrm{CuGaS} 2$ chalcopyrite. In the insets can be seen the absorption spectra for both the semiconductor and the alloys. The grey graphs in the insets are the AMI.5 solar energy absorption spectrum. For the semiconductor the absorption starts close to the fundamental band-gap. For the substituted systems the absorption coefficient starts almost at zero due to low energy transitions and we can clearly notice a huge enhancement of the absorption not only at this low energies but also at all the important solar energy spectrum range. This enhancement seems to be especially great for the Chromium substitution.

The main part of Fig 3 shows the imaginary part of the total dielectric function multiplied by the frequency and the projection of the contribution of the different transitions. We can see how an intra-band IB-IB transition appears first (this transition will not contribute to the photocurrent since the intermediate band is isolated); next the transitions between the VB to the IB which starts and $1 \mathrm{eV}$ for the Titanium system and below 0.5 for the Chromium one. After that we can see the contribution IB-CB. increasing the absorption at around $2.0 \mathrm{eV}$ for $\mathrm{Ti}$ system and $2.5 \mathrm{eV}$ for the Chromium. For the latter system this IB-CB contribution seems to be not very important. The different behavior for the two substituted systems may be due to the fact that the IB is much closer to VB than to $C B$ in the Chromium than in the Titanium alloy. Another appreciable difference is that the intra-band peak is enlarged. probably because the fact that IB of $\mathrm{Cr}$ doped compound is a triplet while that of $\mathrm{TiCu}_{4} \mathrm{Ga}_{3} \mathrm{~S}_{8}$ compound is a doublet. Finally we can see the contributions from the usual transition between VB-CB that starts almost at the same energy in both systems.

2) Spinels and derivatives: For this systems the optical absorption as well as other optical properties has been calculated for the semiconductor as well as for the spinel alloys. The most interesting conclusion is again that with the transition metal we get a significant increase of the absorption of low energy photons compared to that of the semiconductor. This increase occurs at the main region of the solar spectrum as is required for an intermediate band material for photovoltaic purposes. In the insets of Fig. 4 we can see this enhancement by comparing the V-substituted compounds with their corresponding host spinels [12]. In Fig. 4b we also compare the $\operatorname{In}_{2} S_{3}$ optical absorption with available experimental results for this compound. In this case. we show the predicting capabilities of the used approach, since our calculation is in good agreement with the experimental measures of Gillen et al. [13].
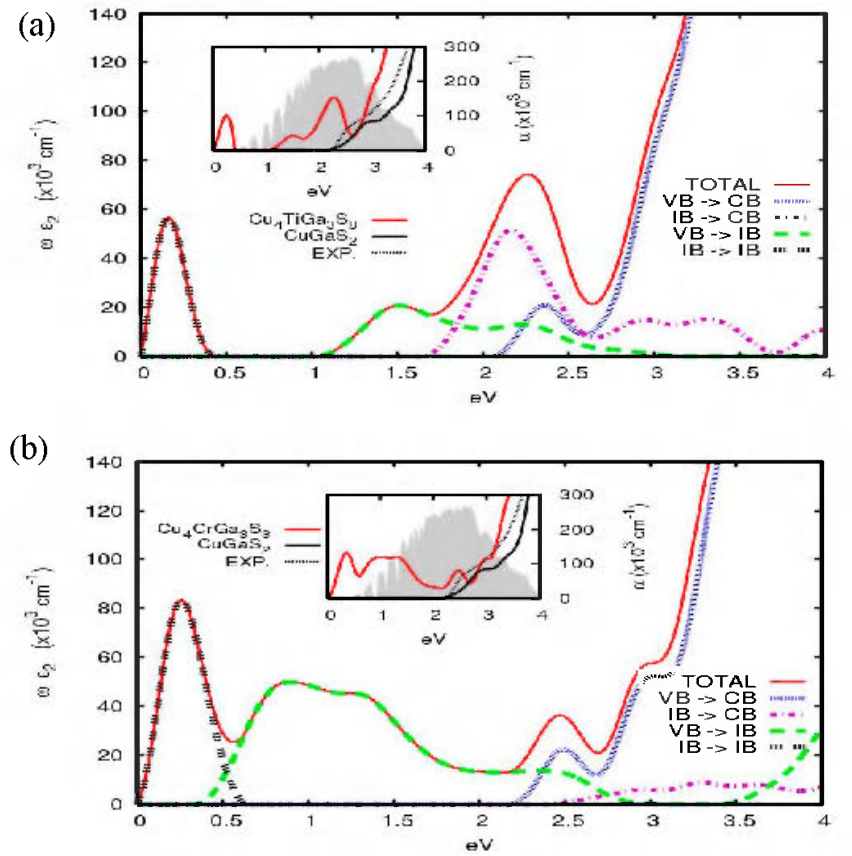

Fig 3. (1)-weighted imaginary part of the dielectric function of $\mathrm{Cu}_{4} \mathrm{Ga}_{3} \mathrm{~S}_{8} \mathrm{M}$; $\mathrm{M}=\mathrm{Ti}$ (a) and $\mathrm{Cr}$ (b). Inset: total absorption coefficient compared to that of the host semiconductor.

Analyzing the frequency-weighted imaginary part of the total dielectric function and its partial contributions to the different transitions we can see, as in chalcopyrites that the intra-band IB-IB transition appears first; next we have the transitions VB-IB which increases the transition around $1 \mathrm{eV}$. Then we have the IB-CB transition increasing mainly the absorption at around $2.5 \mathrm{eV}$; finally the usual one between the VB and the CB. These contributions are shown in Figure 4 and they have a similar shape for the two systems $\left(\mathrm{Mg}_{2} \mathrm{In}_{3} \mathrm{VS}_{8}\right.$ and $\mathrm{In}_{14} \mathrm{~V}_{2} \mathrm{~S}_{24}$ ).

\section{Experimental Results.}

$\mathrm{In}_{2} \mathrm{~S}_{3}$ substituted with Vanadium has been experimentally obtained by a solvothermal method [14]. First the semiconductor was obtained in a similar way. The diffuse reflectance UV-Visible-Near IR (NIR) spectrum measured for the substituted system shows how the different peaks in the 
theoretical calculation have their corresponding signal in the NIR spectrum, validating our calculations. See Fig 5.
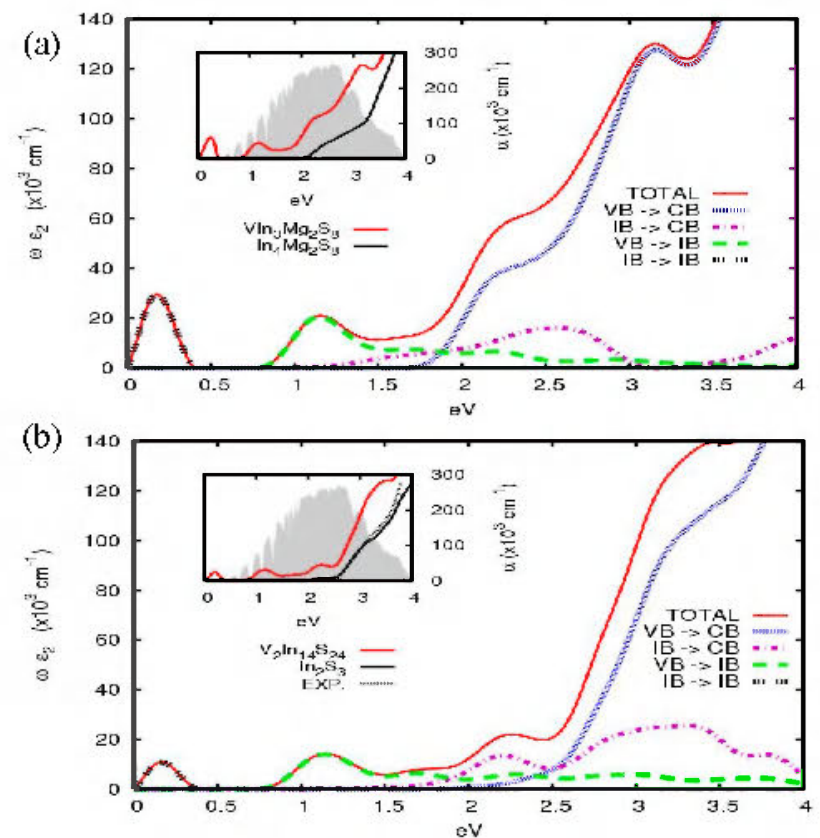

Fig 4. $\omega$-weighted imaginary part of the dielectric function of $\mathrm{Mg}_{2} \ln _{4} \mathrm{~S}_{8} \mathrm{~V}$ (a) and $\operatorname{In}_{14} \mathrm{~S}_{24} \mathrm{~V}_{2}$ (b): Inset: total absorption coefficient of each system compared to that of the host semiconductor.

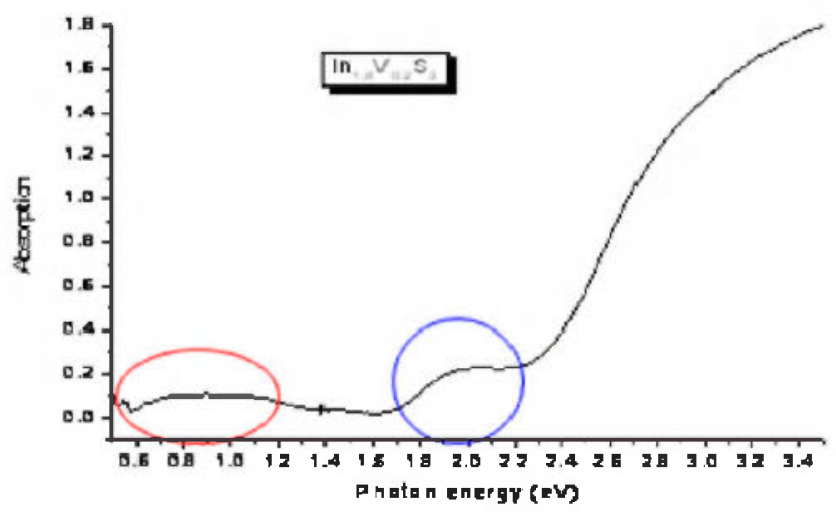

Fig 5: NIR diffuse reflectance spectrum of the substituted vanadium alloy $\left(\operatorname{In}_{14} \mathrm{~S}_{24} \mathrm{~V}_{2}\right)$.

\section{CONCLUSIONS}

We have made DFT calculations for $\mathrm{CuGaS}_{2}, \mathrm{MgIn}_{2} \mathrm{~S}_{4}$ and $\mathrm{In}_{2} \mathrm{~S}_{3}$ systems and substitute one or several of their Ill-type atoms by transition metal atoms. The new compounds present an intermediate band into the usual VB-CB band-gap. Using this IB band, they can absorb sub-band-gap energy photons and are able to obtain a significant increase of the absorption at low energy and in the overall absorption range of the solar spectrum. These materials fulfill the intermediate band photovoltaic material concept being the basis of a new generation of solar cells. In this work we have confirmed the effect of the enhancement in the optical properties of the material resulting from the intermediate transition metal band with experimental results.

\section{ACKNOWLEDGMENT}

We would like to acknowledge the funding from project FULLSPECTRUM (contract. SES-CT-2003-502620) of the $6^{\text {th }}$ European Framework Programme and the from the CALIBAND(MAT2006-10618) project of the Spanish Ministry of Education and Science and from the Community of Madrid NUMANCIA-MA(S-05050/ENE/0310) Research Programme. The author acknowledge the computer resources provided by the Madrid Supercomputing Center (CeSViMa)

\section{REFERENCES}

[1] A. Luque, A. Marti, "Increasing the efficiency of ideal solar cells by photon induced transitions at intermediate levels", Phys. Rev. Lett., Vol. 78, pp .5014-5017. 1997.

[2] P. Palacios, J. J. Fernandez, K. Sanchez, J.C. Conesa, P. Wahnon, "Firstprinciples investigation of isolated band formation in half-metallic $\mathrm{Ti}_{x} \mathrm{Ga}_{1}$ ${ }_{x} \mathrm{P}(x=0.3125-0.25)$. Phys. Rev. B. Vol 73, pp. 085206, 2006.

[3] P. Palacios, K. Sanchez, J.C. Conesa, P. Walınon, "First principles calculation of isolated intermediate bands formation in a transition metaldoped chalcopyrite-type semiconductor". Phys. Stat. Solidi (a) Vol 203 . pp 1395-1401. 2006.

[4] P. Palacios, K. Sanchez, J.C. Conesa, J.J. Fernandez, P. Wahnon, "Theoretical modelling of intermediate band solar cell materials based on metal-doped chalcopyrite compounds". Thin Solid Films, Vol.515. pp. 62806284, 2007

[5] P. Palacios, P. Wahnon, S. Pizzinato, J.C. Conesa, "Energetics of formation of TiGa3As4 and TiGa3P4 intermediate band materials". J. Chem. Phys. Vol. 124, pp. 014711, 2006

[6]Filed Spanish patent application. Ref. nr. ES 200702008. Prioritiy date: July 19 th 2007

[7] G. Kresse, J. Hafner, "Ab initio molecular dynamics for liquid metals", Phys. Rev. B, Vol. 47, RC558, 1993; G. Kresse, J. Furthmuller, "Efficient iterative schemes for $a b$ initio total-energy calculations using a plane-wave basis set ${ }^{2 i}$. Phys. Rev. B. Vol 54, pp. 11169-11186, 1996: G. Kresse, J. Joubert, "From ultrasoft pseudopotentials to the projector augmented-wave method", Phys. Rev. B, Vol. 59. pp. 1758-1775. 1999.

[8] M. Gajdos, K. Hummer, G. Kresse, J. Furthmuller, F. Bechstedt, "Linear optical properties in the projector-augmented wave methodology", Phys. Rev. B. Vol. 73, pp. 045112, 2006.

[9] B. Adolph, J. Furthmuller, F. Bechstedt, "Optical properties of semiconductors using projector-augmented waves", Phys. Rev. B, Vol. 63. pp. 125108, 2001

[10] P. Wahnon, P. Palacios, K. Sanchez, I. Aguilera and J.C. Conesa, "First principles calculation of isolated intermediate bands formation in a transition metal-doped chalcopyrite-type semiconductor", Proceedings $22^{\text {nd }}$ European Photovoltaic Solar Energy Conference, Ed. Wip-Renewable Energies, Munich, 2007, Vol 1,pp 56-59

[11] N. Rampersadh, A. Venter, and D. Billing, "Rietveld refinement of In2S3 using neutron and X-ray powder diffraction data" Phys. B. Vol 350 Suppl. 1 . pp. E383-E385, 2004

[12] P. Palacios, I. Aguilera, K. Sanchez, J.C. Conesa, and P. Walınon, "Transition-metal-substituted indium thiospinels as novel intermediate-band materials: prediction and understanding of their electronic properties", Phys. Rev. Letters., Vol. 101, pp. 046403, 2008

[13] C. Guillen, T. Garcia, J. Herrero, M. T. Gutierrez, F. Briones, "Tailoring growth conditions for modulated flux deposition of $\operatorname{In}_{2} \mathrm{~S}_{3}$ thin films". Thin Solid Films. Vol 451-452, pp. 112-115, 2004

[14] R. Lucena, I. Aguilera, P. Palacios, P. Walmon and J.C. Conesa, "Synthesis and spectral properties of nanocrystalline V-substituted $\operatorname{In}_{2} \mathrm{~S}_{3}$, a novel material for more efficient use of solar radiation" Chem. Mat. Doi: $10.1021 / \mathrm{cm} 801128 \mathrm{~b}(2008)$ in press. 